\title{
New Simulations for lon-Production and Back-Bombardment in GaAs Photo-guns
}

\author{
Josh Yoskowitz* \\ Old Dominion University \\ 5115 Hampton Blvd, Norfolk, VA 23529, USA \\ E-mail: yoskowijejlab.org
}

J. Grames, J. Hansknecht, C. Hernandez-Garcia, G. A. Krafft, M. Poelker, R.

\section{Suleiman}

Thomas Jefferson National Accelerator Facility

12000 Jefferson Ave, Newport News, VA 23606 USA

\section{G. Palacios-Serrano, S. Wijethunga}

Old Dominion University

5115 Hampton Blvd, Norfolk, VA 23529, USA

\section{S. B. Van Der Geer}

Pulsar Physics

Eindhoven, The Netherlands

GaAs-based DC high voltage photo-guns used at accelerators with extensive user programs must exhibit long photocathode operating lifetime. Achieving this goal represents a significant challenge for proposed high average current facilities that must operate at tens of milliamperes or more. Specifically, the operating lifetime is dominated by ion back-bombardment of the photocathode from ionized residual gas. While numerous experiments have been performed to characterize the operating lifetime under various conditions, detailed simulations of the ion backbombardment mechanism that explains these experiments are lacking. Recently, a new user routine was implemented using the code General Particle Tracer (GPT) to simulate electron impact ionization of residual beam line gas and simultaneously track the incident electron, the ejected electron, and the newly formed ion. This new routine was benchmarked against analytical calculations and then applied to experiments performed at the CEBAF injector at the Thomas Jefferson National Accelerator Facility to study the effectiveness of limiting ions from entering the cathodeanode gap using a positively biased anode. These simulations were performed using detailed 3D field maps produced with CST Microwave Studio describing the photo-gun electrostatics. Discussion of the experiment and the application of this new GPT routine to model the experiments will be presented at the workshop.

The 18th International Workshop on Polarized Sources, Targets, and Polarimetry, PSTP2019

23-27 September, 2019

Knoxville, Tennessee

\footnotetext{
*Speaker.
} 


\section{Introduction}

One of the biggest challenges in creating sustained, high current electron beams in GaAs-based DC high voltage photo-guns is the mitigation of ion back-bombardment [1,2,3]. The electron beam can ionize residual gas within the cathode-anode gap and adjacent beam line. Positive ions formed in the cathode-anode gap are accelerated towards the photocathode. In addition, some of the ions created downstream of the anode can reach the photocathode if the anode is grounded. When ions strike the photocathode, X-rays can be produced, leading to unwanted photo-emission from the edges of the photocathode. These electrons can strike the vacuum chamber, causing gas desorption and more ion back-bombardment of the photocathode. These deleterious ion effects lead to quantum efficiency (QE) degradation and a decrease in the photocathode charge lifetime. Figure 1 below shows the ion back-bombardment process in the CEBAF photo-gun.

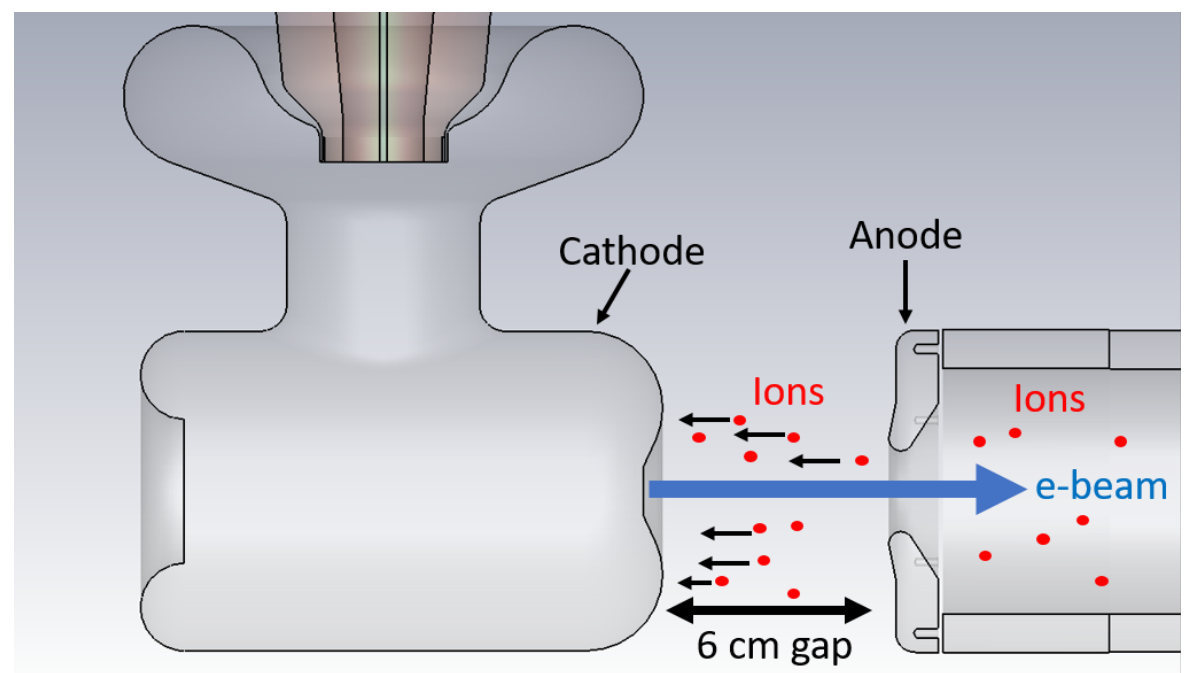

Figure 1: Diagram of the CEBAF photo-gun showing the locations of the cathode and anode electrodes. The electron beam ionizes residual gas. Ions produced within the anode/cathode gap are accelerated towards the negative potential of the cathode. Ions downstream of the anode are repelled when the anode is positively biased.

The approach taken to hinder ion back-bombardment and increase the lifetime of the photocathode was to positively bias the anode. When the anode is biased positively, ions downstream of the anode that would otherwise strike the photocathode are instead repelled away from it. This technique has been described in [4] and experiments involving a biased anode have been performed at Jefferson Lab [5, 6, 7], though detailed simulations of the ion back-bombardment mechanism in the CEBAF photo-gun with a biased anode have not been done prior to this work.

\section{Ionization Theory}

An electron may produce a positive ion if it scatters from residual gas atom or molecule and knocks out one of its electrons. The incident electron is called the primary electron and the ejected electron is called the secondary electron. This process is called electron impact ionization and is shown in Figure 2. 


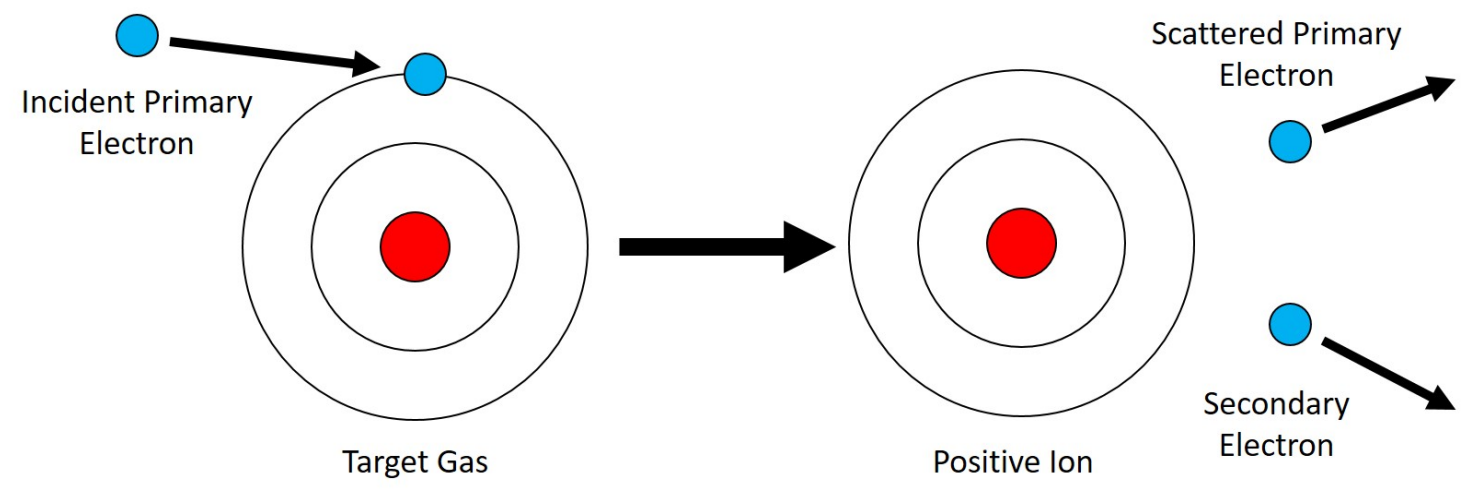

Figure 2: Diagram of electron impact ionization: a beam electron knocks an electron out of a target gas atom or molecule, resulting in a positive ion, secondary electron, and scattered electron.

\subsection{Ionization Cross Section and Ion Production Rate}

The probability that an electron will ionize residual gas upon collision is given by the ionization cross section $\sigma[8]$ :

$$
\begin{aligned}
\sigma_{i}\left[\mathrm{~m}^{2}\right] & =\frac{1.872 \times 10^{-24} A_{1}}{\beta_{e}^{2}} f\left(T_{e}\right)\left[\ln \left(7.515 \times 10^{4} A_{2} \beta_{e}^{2} \gamma^{2}\right)-\beta_{e}^{2}\right] \\
f\left(T_{e}\right) & =\frac{I_{i}}{T_{e}}\left(\frac{T_{e}}{I_{i}}-1\right) \\
A_{1} & =M^{2} \\
A_{2} & =\frac{e^{\frac{C}{M^{2}}}}{7.515 \times 10^{4}}
\end{aligned}
$$

Here, $\beta_{e}$ and $\gamma$ are relativistic factors for the primary electron, $A_{1}$ and $A_{2}$ are derived from empirical constants given by Rieke and Prepejchal that depend on the gas species [9], and $f\left(T_{e}\right)$ is a function for fitting $\sigma$ at low energies, i.e. when the primary electron kinetic energy $T_{e}$ is close to the ionization energy $I_{i}$ of the target atom or molecule. Only electrons with a kinetic energy greater than the ionization energy $I_{i}$ are able to ionize.

Consider an electron beam with current $I_{e}$ moving through gas with density $\rho_{i}$. In a given amount of time $\Delta t$, the electrons will move a distance $l$ through the gas. The average ion production rate is given by

$$
\frac{N_{i}}{\Delta t}=\rho_{i} \sigma_{i} l \frac{I_{e}}{e}
$$

Dividing both sides by $l$, we can write an equation for the ion production rate per unit length:

$$
\frac{N_{i}}{\Delta t \cdot l}=\rho_{i} \sigma_{i} \frac{I_{e}}{e}
$$

If we know the number of electrons per unit time passing through the gas instead of the electron current, we can rewrite (2.2) as

$$
\frac{N_{i}}{\Delta t}=\rho_{i} \sigma_{i} l \frac{N_{e}}{\Delta t}
$$


The average number of ions $N_{i}$ produced over a given distance $l$ is then

$$
N_{i}=\rho_{i} \sigma_{i} l N_{e}
$$

Figure 3 shows the ionization cross section and ion production rate per for $\mathrm{H}_{2}$, the dominant gas species in the CEBAF photo-gun and beam line, as a function of beam energy and Table 1 below shows calculated values of the ionization cross section and ion production rate per unit length of $\mathrm{H}_{2}$ gas for various beam energies. Since the ionization cross section is much larger for low energy electrons compared to high energy electrons, the electron beam creates more ions near the cathode than near the anode.
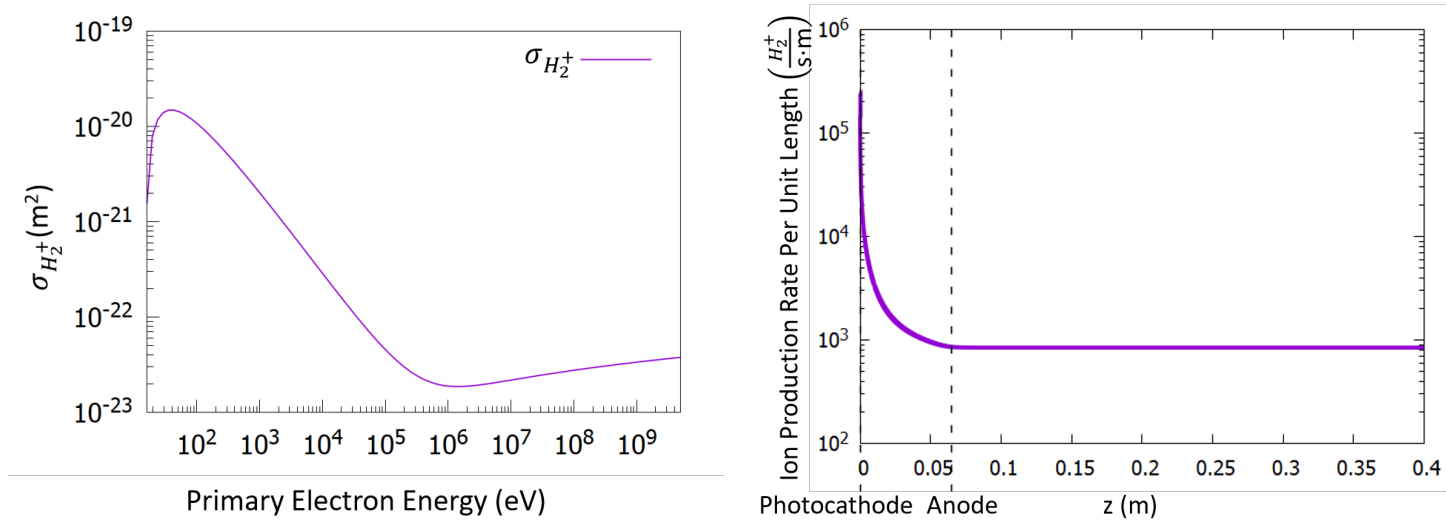

Figure 3: Left: $\log -\log$ plot of the ionization cross section as a function of primary electron energy. Right: plot of the ion production rate within the CEBAF photo-gun and beam line for $\mathrm{H}_{2}$ gas assuming a density of $\rho=3.52 \times 10^{10} \mathrm{~m}^{-3}\left(10^{-12}\right.$ Torr $)$ and a beam current of $I_{e}=100 \mu \mathrm{A}$.

\begin{tabular}{|c|c|c|}
\hline Primary Electron Energy $(\mathrm{keV})$ & $\sigma_{H_{2}^{+}}\left(\mathrm{m}^{2}\right)$ & $\frac{d N_{H_{2}^{+}}}{d t}\left(\frac{1}{l}\right)\left(\frac{H_{2}^{+}}{\mathrm{s} \cdot \mathrm{m}}\right)$ \\
\hline 0.1 & $1.1 \times 10^{-20}$ & $2.4 \times 10^{5}$ \\
\hline 1 & $2.0 \times 10^{-21}$ & $4.4 \times 10^{4}$ \\
\hline 10 & $2.9 \times 10^{-22}$ & $6.3 \times 10^{3}$ \\
\hline 100 & $4.6 \times 10^{-23}$ & $1.0 \times 10^{3}$ \\
\hline
\end{tabular}

Table 1: Calculated values of the ionization cross section and ion production rate per unit length for $\mathrm{H}_{2}$ gas assuming a density of $\rho=3.52 \times 10^{10} \mathrm{~m}^{-3}\left(10^{-12} \mathrm{Torr}\right)$ and a beam current of $I_{e}=100 \mu \mathrm{A}$.

\subsection{Ion Production Rate within the CEBAF Photo-gun}

A 3D electric field map of the CEBAF photo-gun was created using a CST model for the cathode biased at $-130 \mathrm{kV}$ and either a $0 \mathrm{~V}$ or a $1 \mathrm{kV}$ anode. The plots in Figure 4 show the $z$-component of the electric field of the CEBAF photo-gun as a function of $z$ along the central axis for the anode grounded (top) and biased at $+1 \mathrm{kV}$ (bottom). For both anode bias configurations, ions produced within the cathode-anode gap will be accelerated toward the photocathode where they can degrade photocathode QE. For the grounded anode configuration, some ions produced downstream of the anode will strike the photocathode and it is believed these ions are much more 
damaging than ions created within the cathode-anode gap because they strike the photocathode with maximum energy. But as illustrated in the bottom plots, if the anode were positively biased, ions downstream of the anode would be repelled away.
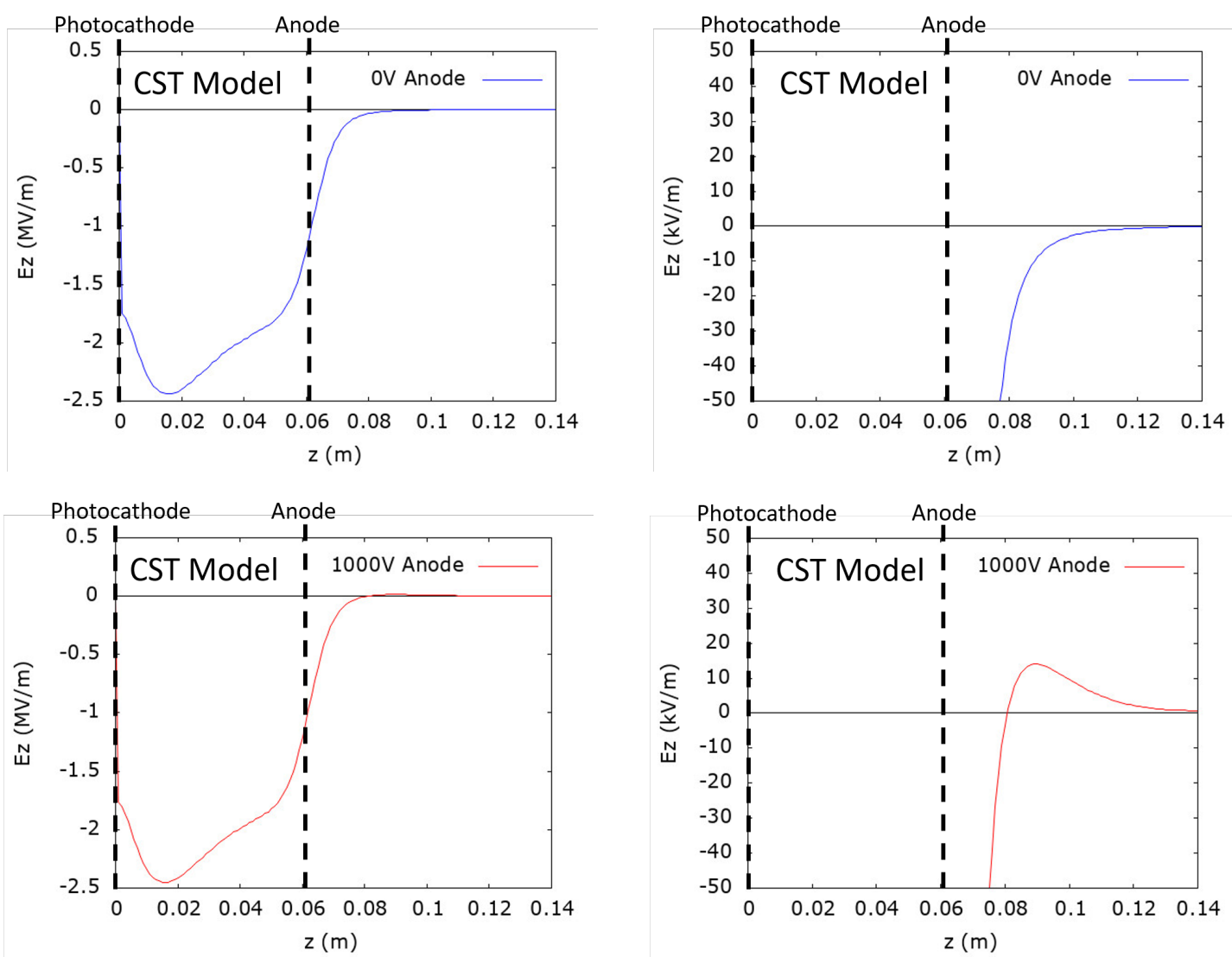

Figure 4: Plots of $E_{z}$ as a function of $z$ up to $0.14 \mathrm{~m}$ downstream of the photocathode for a $-130 \mathrm{kV}$ cathode and $0 \mathrm{~V}$ anode (top) and $+1 \mathrm{kV}$ anode (bottom). The vertical scale of the left plots show the entire range of the electric field while the vertical scale of the right plots are set to $\left|E_{z}\right| \leq 50 \frac{\mathrm{kV}}{\mathrm{m}}$ for easier visualization of $E_{z}$ due to the anode bias.

\section{Ion Back-Bombardment Simulations Using GPT}

\subsection{Initial Particle Distribution}

To interpret the measurements performed during the run period, preliminary ion simulations were performed using the simulation package General Particle Tracer (GPT) [10]. To generate the initial ion distribution in the GPT simulations, a $0.2 \mathrm{pC}$ electron bunch with a $1 \mathrm{~mm}$ RMS transverse size and 5 ps RMS bunch length (corresponding to a typical CEBAF average current of $10 \mu \mathrm{A}$ ) was tracked $0.4 \mathrm{~m}$ downstream of the photocathode. This simulation used a 3D electric field map of the CEBAF photo-gun created using CST Studio Suite software [11] and neglected any space charge effects. Due to the geometry of the cathode electrode, the electrons receive a vertical kick downward after leaving the photocathode [12]. 
By dividing the simulation into many timesteps and noting the positions of the primary electrons at each timestep, a convex volume that surrounds all positions where the electrons in the electron bunch pass can be created via a 3D convex hull. Since ions can only be created where the electron bunch passes, the convex hull was uniformly filled with $10^{5}$ macro-particles and was used as the initial ion macro-particle distribution for the GPT simulations. The number of ions each macro-particle represents was weighted using equation (2.5) and is based on the kinetic energy of the electron bunch at the macro-particle's initial location. Hydrogen gas is the predominant gas species in the baked vacuum systems. Thus, for simplicity, it is assumed that only $\mathrm{H}_{2}^{+}$ions are created with the typical CEBAF photo-gun pressure of $10^{-12}$ Torr. The kinetic energy of each ion is equal to the thermal energy at room temperature $(293.15 \mathrm{~K})$, which corresponds to $T_{H_{2}^{+}}=38 \mathrm{meV}$, and the direction of each ion after ionization is random. Figure 5 shows the initial spatial distribution of ion macro-particles and a plot of the total number of ions as a function of distance $z$ from the photocathode.

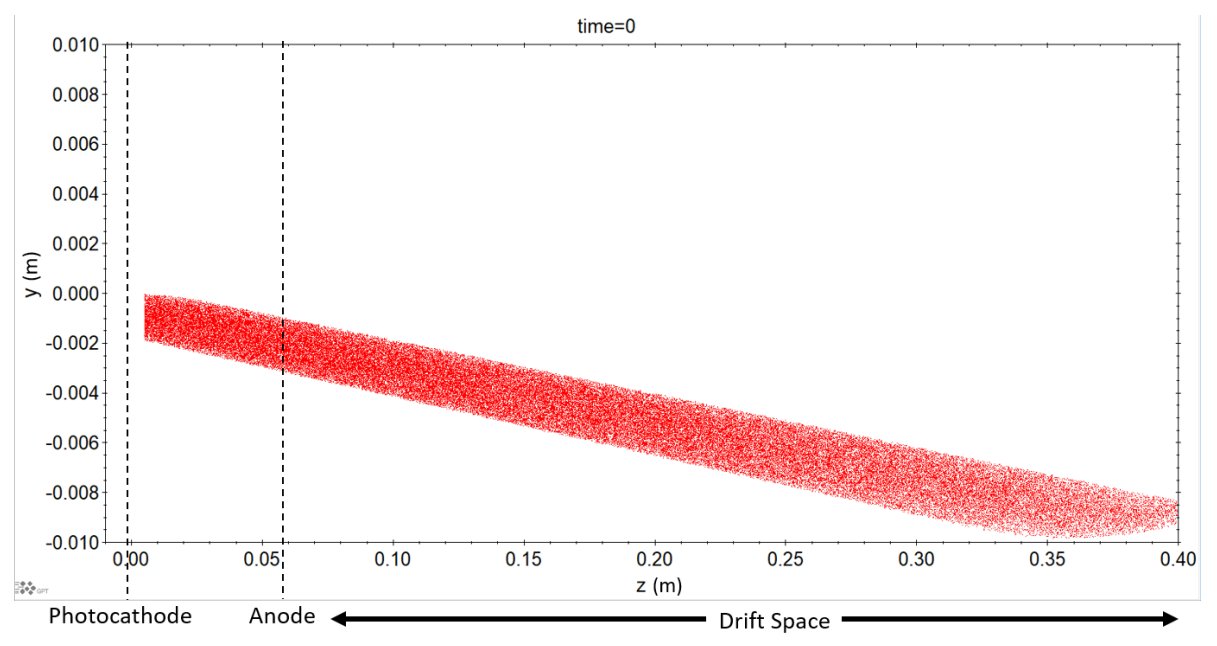

Figure 5: Side-view of the initial ion distribution for GPT simulations. Each red dot represents an individual ion macro-particle.

\subsection{GPT Simulations}

Once the initial ion macro-particle distribution was defined, GPT tracked the movement of ions based on their initial kinetic energy and the electric field of the CEBAF photo-gun for a total of $3 \mu \mathrm{s}$. During the simulations, GPT used a 3D electric field map of the CEBAF photo-gun using a CST model, assuming a $-130 \mathrm{kV}$ cathode and a $0 \mathrm{~V}$ anode for the grounded anode configuration or a $1 \mathrm{kV}$ anode for the biased anode configuration. Because the anode bias has a negligible effect on the initial ion distribution, the same initial distribution was used for simulations of both configurations.

Figure 6 shows the distribution of the ions for the GPT simulations of the grounded and biased anode after $2.25 \mu \mathrm{s}$. In the grounded anode simulation, we see that ions that are within the cathodeanode gap accelerate towards the photocathode and get deflected upward due to the optics of the cathode-anode geometry. In the biased anode simulation, ions within the cathode-anode gap behave similarly to the grounded anode case. However, ions downstream of the anode are repelled away 
and prevented from passing through the anode and reaching the photocathode. It must be stressed that these simulations are very preliminary and are currently being improved upon to increase accuracy and precision.
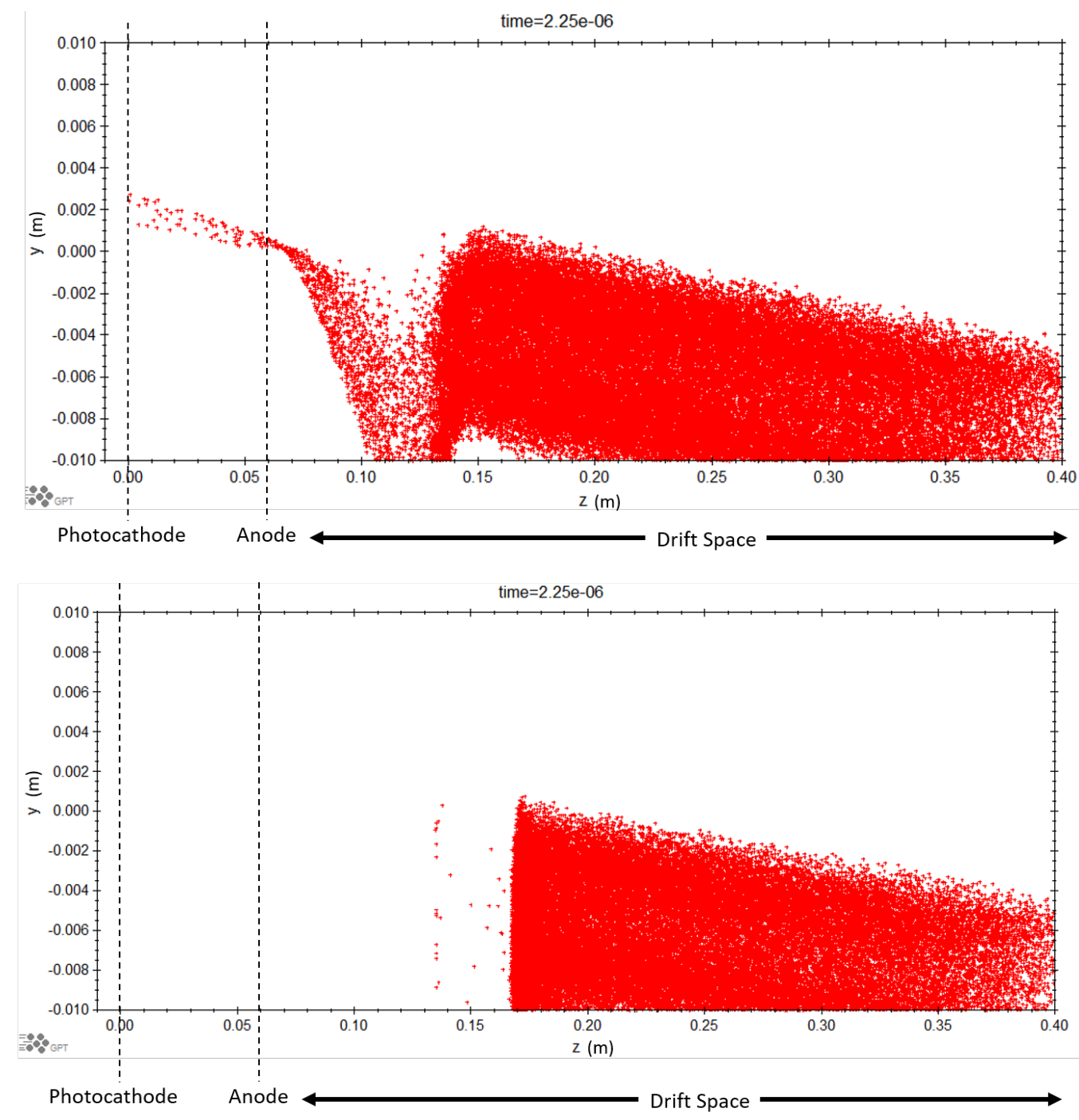

Figure 6: GPT simulations of the CEBAF photo-gun after $2.25 \mu$ starting with the initial ion distribution from Figure 5, assuming a $-130 \mathrm{kV}$ cathode and a $0 \mathrm{~V}$ anode (top) or a $1 \mathrm{kV}$ anode (bottom).

\section{Biased Anode Experiments during 2019 CEBAF Summer Run}

\subsection{Experimental Setup}

The CEBAF photo-gun is constructed with an electrically isolated anode, which allows the anode to be either grounded or biased via a UHV compatible high voltage feedthrough. Figure 7 shows the CEBAF photo-gun with the anode feed-through for biasing. Providing a small, positively biased, ion-repelling field downstream of the photo-gun proved relatively innocuous to implement. This required modifying the downstream 10" flange that supports the anode with a UHV compatible 
$10 \mathrm{kV}$ high voltage feed-through. This modification provides a thin stand-off from the internal fixture of the gun chamber for the anode itself and connects a wire strung with ceramic bushings to complete the electrically isolated circuit. Based on calculations and the electrostatic model, only a small voltage of $1-2 \mathrm{kV}$ is required to provide a sufficient barrier to repel ions with energies running from thermal velocities to the energies gained by the trapping potential of the electron beam. Additionally, measurements characterizing the electron beam (not reported here) indicate no discernible difference in the electron beam size, bunch timing, or energy spread when the anode is biased or grounded.

Between June 15th and September 9th of 2019, the CEBAF program delivered three electron beams to Halls A, B and C with typical total average currents of $70-100 \mu \mathrm{A}$. Figure 7 shows a plot of the gun current and total charge extracted as a function of time for the entire run period. The anode bias was grounded at the beginning of the run period and then alternated between $1 \mathrm{kV}$ and $0 \mathrm{~V}$ every 2-3 weeks, which corresponds to about 50-60 $\mathrm{C}$ of charge extracted from the photocathode for each anode bias configuration.
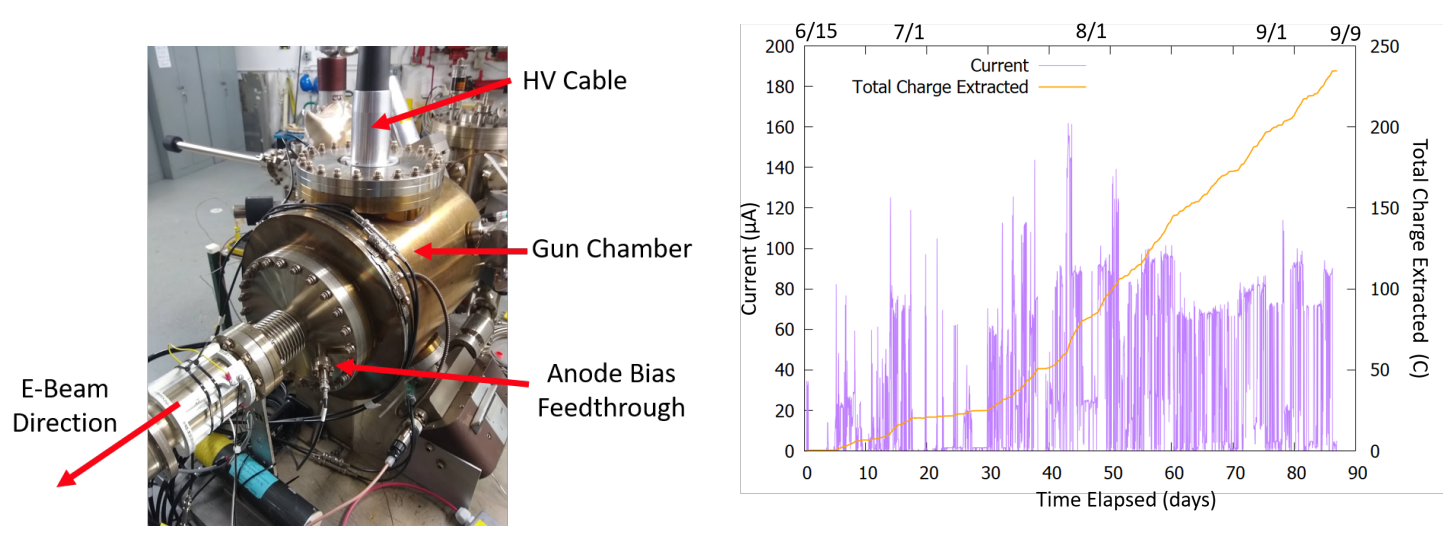

Figure 7: Photo of the CEBAF photo-gun (left) and plot of the electron current extracted from the photocathode over the run period (right).

\subsection{QE Measurements}

QE measurements of the photocathode using each of the three lasers were made every morning for the entire run period. During a QE measurement, the electron beam is stopped briefly, and each laser sequentially illuminates the photocathode to produce a small amount of electron current $I$ that is measured by a picoammeter. Using each laser's wavelength $\lambda$ and power $P$ (as measured by a power meter), the $\mathrm{QE}$ of the photocathode at the laser spot was calculated using the following formula:

$$
\begin{aligned}
\mathrm{QE} & =\frac{h c}{e} \frac{I}{\lambda P} \\
\mathrm{QE}(\%) & =\frac{124 * I(\mathrm{~mA})}{\lambda(\mathrm{nm}) P(W)}
\end{aligned}
$$


Here, $h$ is the Planck constant, $c$ is the speed of light in vacuum, and is the elementary charge. Figure 8 shows all QE measurements for each laser as a function of total charge extracted from the photocathode.

For each anode configuration run period, the QE measurements for each laser were fit with an exponential decay function of the form:

$$
\mathrm{QE}=\mathrm{QE}_{0} \exp \left(-\frac{q}{\tau}\right)
$$

Here, $\tau$ is the charge lifetime of the photocathode: the amount of charge $q$ extracted from the photocathode before the QE drops to 1/e of itself. Table 2 lists charge lifetime values for each laser and anode bias configuration. To quantify the improvement with the anode biased, Table 3 shows the ratio of the lifetimes between regions 1 and 2 and between regions 3 and 4 for each laser.

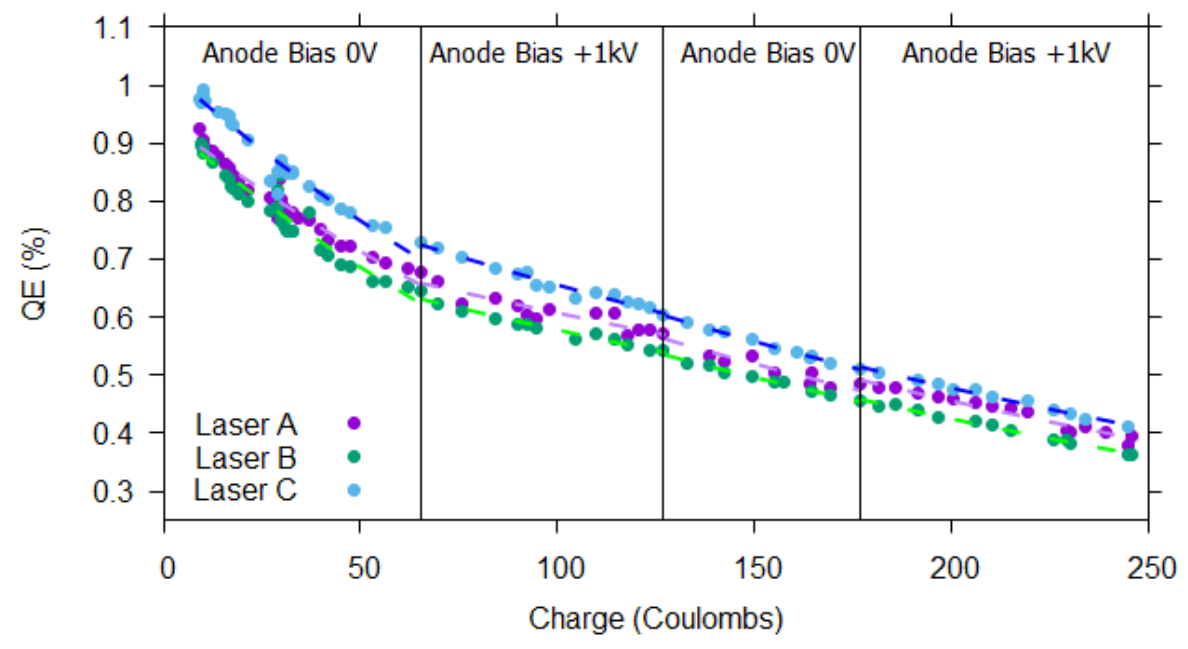

Figure 8: Plot of QE measurements for each laser as a function of the total charge extracted from the photocathode. The plot is divided into four regions that correspond to each anode bias configuration. The dots represent QE measurements and the dashed lines represent exponential decay fits to determine the charge lifetime of each laser in each region. All lasers operate at the same wavelength and are intended to illuminate the same laser spot, thus the same results are expected for each laser.

\begin{tabular}{|r|r|r|c|c|}
\hline Region & Anode Bias (V) & Laser A Lifetime (C) & Laser B Lifetime (C) & Laser C Lifetime (C) \\
\hline 1 & 0 & $181 \pm 8$ & $161 \pm 9$ & $169 \pm 7$ \\
\hline 2 & 1000 & $424 \pm 53$ & $392 \pm 23$ & $346 \pm 16$ \\
\hline 3 & 0 & $288 \pm 39$ & $304 \pm 14$ & $346 \pm 16$ \\
\hline 4 & 1000 & $303 \pm 18$ & $302 \pm 9$ & $314 \pm 11$ \\
\hline
\end{tabular}

Table 2: Charge lifetime values for each anode bias configuration and for each laser. Error values correspond to the fit errors. 


\begin{tabular}{|c|c|c|c|}
\hline Improvement Ratio & Laser A & Laser B & Laser C \\
\hline$\tau_{2} / \tau_{1}$ & $2.34 \pm 0.31$ & $2.43 \pm 0.20$ & $2.05 \pm 0.13$ \\
\hline$\tau_{3} / \tau_{2}$ & $0.68 \pm 0.13$ & $0.78 \pm 0.06$ & $1.00 \pm 0.07$ \\
\hline$\tau_{4} / \tau_{3}$ & $1.05 \pm 0.16$ & $0.99 \pm 0.05$ & $0.91 \pm 0.05$ \\
\hline
\end{tabular}

Table 3: Charge lifetime improvement ratios between successive anode bias configurations.

A charge lifetime improvement ratio $>1$ indicates improved performance compared to the previous configuration and a ratio $<1$ indicates degraded performance. The first and second rows of ratios in Table 3 illustrate an unmistakable benefit of applying a bias voltage to the anode: to repel downstream ions from the photo-gun. It is noteworthy that the relative improvement in the charge lifetime with the anode biased lessens over the course of the study. This interesting feature may be explained in part by: (a) a gradual improvement of charge lifetime independent of the applied bias, which is often observed following a brief reduction in QE after photocathode activation when weakly bound states are first removed; (b) the relative exposure of the illuminated photocathode area to ions that are within the cathode-anode gap or downstream of it; or (c) the anode bias power supply was found faulted at the end of the run period, which suggests that it may have simply stopped working during the last period.

\subsection{QE Scans of the photocathode}

To gauge the ion bombardment of the photocathode, QE scans over the entire photocathode active area (not just at the laser spot) were taken before and after the run period, as shown in Figure 9. During a QE scan, one laser is scanned over the surface of photocathode in a grid of points covering the active area. At each point, the QE is calculated using equation (4.1). The QE measurements are then interpolated to create smooth contour plots of the $\mathrm{QE}$ in the vicinity of the active area. Over the course of the run period, these scans indicate the entire active area experienced QE degradation with the highest amount of degradation at the laser spot.
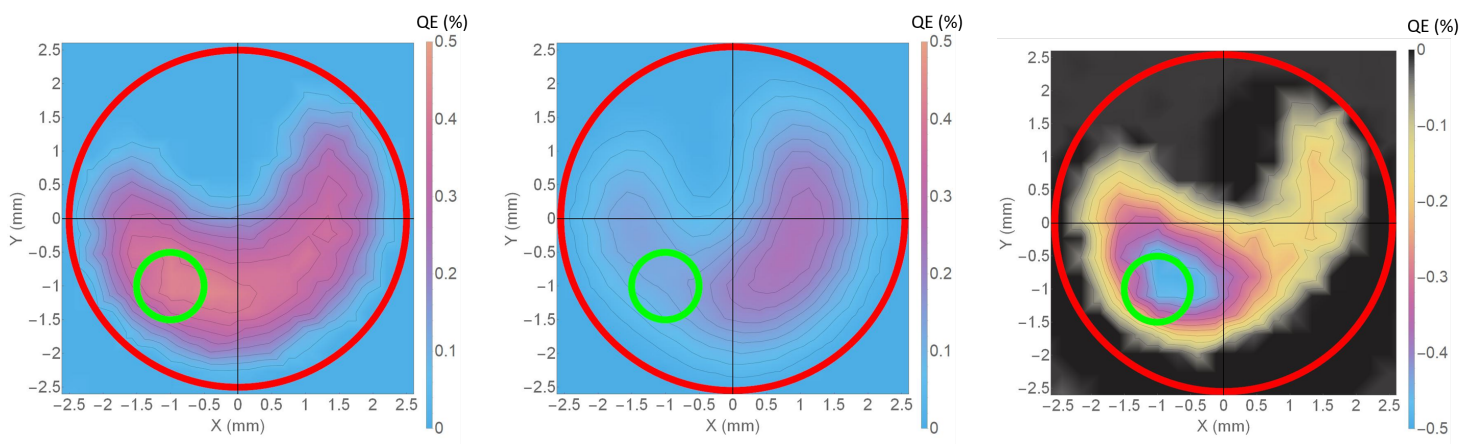

Figure 9: QE scans of the photocathode before (left) and after (middle) the 2019 CEBAF Summer run. The QE map at the right shows the difference between the two QE scans. The red circles denote the 5mmdiameter photocathode active area and the green circles denote the $1 \mathrm{~mm}-\mathrm{FWHM}$ laser spot.

\section{Discussion}

The simulations of ion tracking and back-bombardment of the photocathode in the CEBAF gun 
are new developments in explaining QE degradation during high voltage beam operation. The application of the tracking program General Particle Tracer and inclusion of detailed 3D field profiles computed by CST Studio Suite demonstrate, as in Figure 6, the utility for sophisticated simulations to model, test and benchmark ion damage. This model of ion back-bombardment as a function of the initial conditions is currently under development to improve both accuracy and precision within the GPT simulations. However, these simulations do suggest two points for exploration with more detailed simulations: (a) ions that are accelerated towards the photocathode strike the photocathode above the laser spot with a damage pattern similar to the spatial reduction in QE (see Figure 9) and (b) the biased anode may be effective at repelling ions downstream of the anode that would otherwise strike the photocathode. These will help to explain the 1.5-2 increase in QE lifetime with biased anode configuration (see Figure 8 and Tables 2-3).

\section{Ongoing and Future Work}

A biased anode has been implemented at the CEBAF injector at Jefferson Lab. Its first operation has demonstrated the capability to improve the photocathode charge lifetime and meaningfully extend the useful amount of time Users may receive beam without intervention. The evolution of the improvement with operation is not well understood, but a focus of ongoing research studies.

Simulations using General Particle Tracer are being performed to provide insight into the dynamics of ion back-bombardment. The development of a new GPT custom element that includes electron impact ionization is underway to provide realistic dynamical simulations in real time, a feature not currently supported in GPT. As the custom element is developed, there are plans to benchmark against the program IBSimu [13]. Anticipated improvements to the GPT custom element are the inclusion of multiple gas species, secondary electron production, neutral production, and importantly self-consistent space charge effects of the beam potential. The custom element will be used to create more realistic simulations of the CEBAF photo-gun and help to explain the evolution of charge lifetime under various conditions, including the biased anode.

\section{Acknowledgments}

This material is based upon work supported by the U.S. Department of Energy, Office of Science, Office of Nuclear Physics under contract DE-AC05-06OR23177. This project has received funding from the European Union's Horizon 2020 research and innovation programme under grant agreement No 824093.

\section{References}

[1] K. A. H. G. Andreson et al., Operating experience with the mami polarized electron source, in Proceedings of the Workshop on Photocathodes for Polarized Electron Sources for Accelerators, D. S. M. Chatwell, J. Clendenin T. Maruyama, ed., SLAC-432, (Stanford, CA), pp. 2-12, 1993.

[2] K. Aulenbacher, Polarized beams for electron accelerators, The European Physical Journal Special Topics 198 (2011) 361. 
[3] J. Grames, P. Adderley, J. Brittian, D. Charles, J. Clark, J. Hansknecht et al., Ion back bombardment of GaAs photocathodes inside dc high voltage electron guns, in Proceedings of the 2005 Particle Accelerator Conference, pp. 2875-2877, May, 2005, DOI.

[4] E. Pozdeyev, Ion trapping and cathode bombardment by trapped ions in dc photoguns, Phys. Rev. ST Accel. Beams 10 (2007) 083501.

[5] C. Hernandez-Garcia, P. Adderley, B. Bullard, J. Benesch, J. Grames, J. T. Yoskowitz et al., Compact $-300 \mathrm{kV}$ dc inverted insulator photogun with biased anode and alkali-antimonide photocathode, Phys. Rev. Accel. Beams 22 (2019) 113401.

[6] J. Grames, P. Adderley, J. Brittian, J. Clark, J. Hansknecht, D. Machie et al., A biased anode to suppress ion back-bombardment in a dc high voltage photoelectron gun, AIP Conference Proceedings 980 (2008) 110 [https: / /aip.scitation.org/doi/pdf/10.1063/1.2888075].

[7] J. Grames, R. Suleiman, P. A. Adderley, J. Clark, J. Hansknecht, D. Machie et al., Charge and fluence lifetime measurements of a dc high voltage GaAs photogun at high average current, Physical Review Special Topics - Accelerators and Beams 14 (2011).

[8] M. Reiser, Theory and Design of Charged Particle Beams. Wiley VCH Verlag GmbH, 2008.

[9] F. F. Rieke and W. Prepejchal, Ionization cross sections of gaseous atoms and molecules for high-energy electrons and positrons, Physical Review A 6 (1972) 1507.

[10] General Particle Tracer, 2019.

[11] Computer Simulation Technology, 2019.

[12] G. Palacios-Serrano, F. Hannon, C. Hernandez-Garcia, M. Poelker and H. Baumgart, Electrostatic design and conditioning of a triple point junction shield for a -200 kv dc high voltage photogun, Review of Scientific Instruments 89 (2018) 104703

[https://doi.org/10.1063/1.5048700].

[13] T. Kalvas, O. Tarvainen, T. Ropponen, O. Steczkiewicz, J. Ärje and H. Clark, Ibsimu: A three-dimensional simulation software for charged particle optics, Review of Scientific Instruments 81 (2010) 02B703 [https://doi.org/10.1063/1.3258608]. 